\title{
Management of Spine Trauma and Deformity: An Orthopaedic Perspective
}

Charles R. Clark, M.D.

Associate Professor of Orthopaedic Surgery, University of Iowa College of Medicine, Iowa City, Iowa 52242, U.S.A.

\section{Summary}

This presentation concerns orthopaedic surgery and discusses the present state of art and possible future developments pertaining to the management of injury of the spine and other deforming conditions. It briefly highlights some of the recent advances in the area of neuroradiographic evaluation, closed management and surgical management of traumatic and other deforming conditions of the spine.

Key words: Spinal trauma; Spinal deformity.

\section{Introduction}

There has been a tremendous evolution in the management of traumatic and deforming conditions of the spine. The orthopaedic surgeon plays a major role in managing these conditions. This paper will discuss some of the current and future trends in the management of spinal trauma and deformity. It will also stress the dynamic relationship that the orthopaedic surgeon has with his colleagues including the neurosurgeon, radiologist, anaesthesiologist, and emergency room physician.

\section{Spinal trauma}

Injury to the spinal cord remains a major problem. Reliable estimates show that there are probably upward of 10000 spinal cord injuries that result in paraplegia or quadriplegia each year in the U.S.A. and that there are probably in the neighbourhood of 200000 paraplegic and quadriplegic patients presently living in this country (Pierce et al., 1977). Problems with the management of spinal cord injuries have arisen in several areas. These have occurred either because of improper handling of the patient at the scene of an accident, improper techniques for transporting a patient to the hospital, missed diagnosis in the emergency suite or improper treatment once the proper diagnosis has been established.

Great strides have been made in each of these areas. Emergency medical technicians now receive training in the proper management of spinal injury 


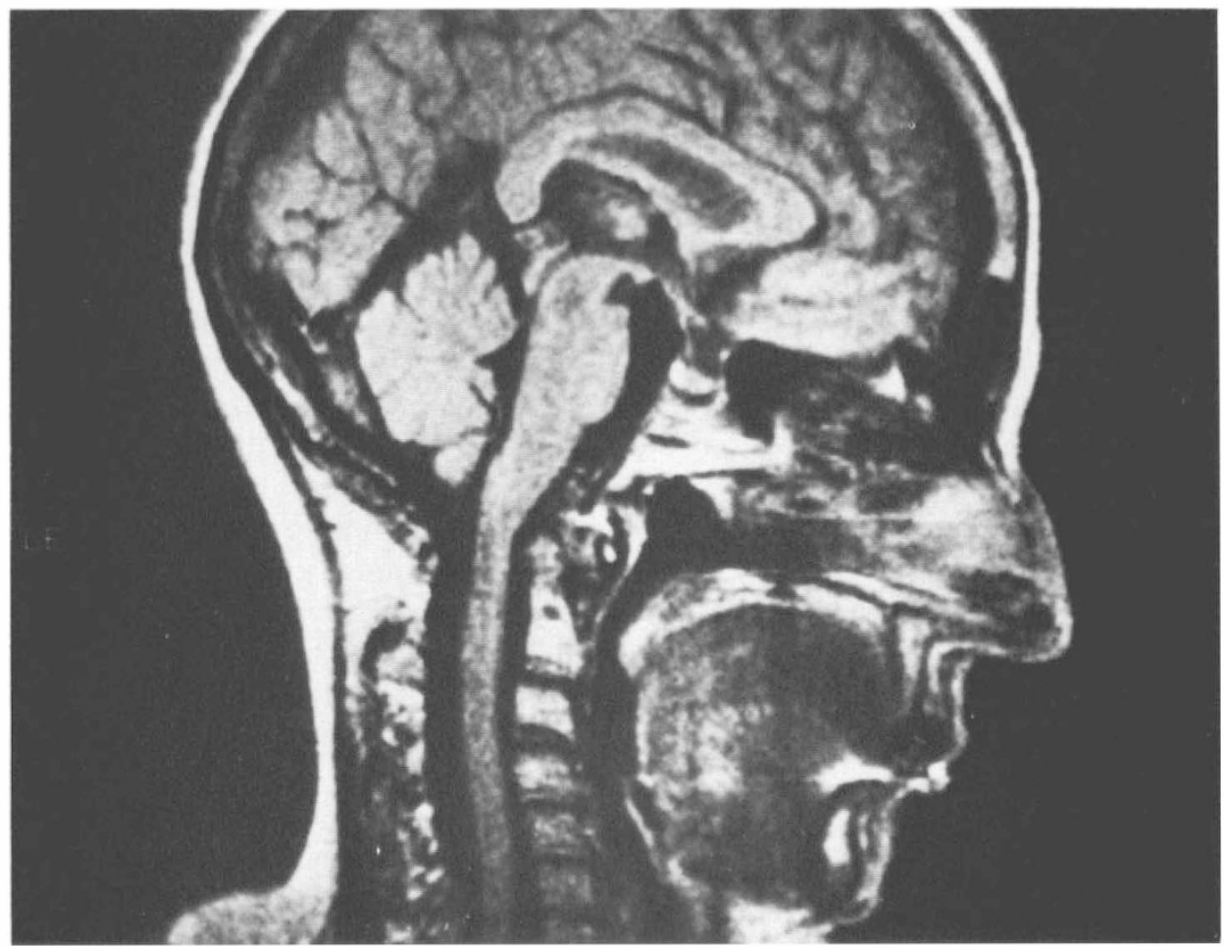

Figure Sagittal magnetic resonance image of the craniovertebral junction demonstrating atlantoaxial subluxation in a patient with rheumatoid arthritis. Note the presence of soft tissue around the dens and the increased pre-dental interval.

patients. The use of spine boards and rigid cervical immobilisation for transport of the injured have become standard procedure in many parts of this country. Emergency room physicians have become keenly aware of the possibility of spinal injury in the multiply traumatised patient as well as the unconscious and/or head injured patient. Routine spine radiographs are obtained in most centres on the multiply injured patient. The availability of advanced diagnostic studies in the emergency room has greatly improved in recent years. Conventional tomography, computerised tomography, myelography and magnetic resonance imaging studies are present in most centres. The availability of these studies has led to accurate and timely diagnosis of the extent of spinal injury and consequently the prompt institution of appropriate treatment. The availability of fluoroscopic imaging in the emergency room has also increased our ability to perform closed reductions of many cervical injuries including unilateral or bilateral facet subluxations or dislocations and rotatory subluxations of the atlantoaxial complex. Skeletal traction is applied in the emergency room. Patients are taken directly to the radiology suite and closed reductions may be attempted with the patient awake, monitoring the radiologic status with an image intensifier and the neurologic status by examination.

Magnetic resonance imaging has been a major advance in the management of both traumatic and other deforming conditions of the spine. There have been many clinical series describing the role of magnetic resonance imaging in 
managing neurologic disease (Spetzler et al., 1985). Magnetic resonance imaging is particularly useful in assessing the craniovertebral junction (Kean et al., 1983). This is an area which is difficult to visualise with conventional radiographic studies. We have found this study to be most useful in patients with severe rheumatoid destruction of the upper cervical spine (Figure). In many cases we have been able to avoid the use of invasive myelographic studies and yet accurately evaluate the degree of instability and cord compression at the craniovertebral junction. By accurately assessing the anatomic level and degree of neurologic compromise the magnetic resonance image, along with other advanced imaging techniques including myelography with non-ionic contrast material have greatly increased our ability to manage spinal conditions effectively and timely.

Cervical orthoses and skeletal pin fixation have undergone tremendous development in recent years. Gardner-Wells tongs are a convenient traction apparatus which may be applied by the surgeon in the emergency room and are very useful for stabilising the acutely injured spine. These tongs have a built-in spring mechanism which allows the surgeon to accurately determine the amount of tension applied by the pin to the skull and can easily be applied by one person in the emergency room.

The halo device remains the most effective closed method of stabilising the spine. There has been tremendous research and development in the area of pin design, placement, and appropriate amount of pressure to be applied (Garfin et al., 1985). Further research is also being done on the best means of attaching the halo device to the body and there has been a great deal of effort designing new types of vests which securely fasten the halo apparatus to the trunk, yet are not unduly cumbersome to the patient (Krag et al., 1986). This is indeed a very important concern in the spinal cord injury patient with insensate skin or the frail elderly patient with myelopathy as is the case with many advanced rheumatoid patients.

\section{Surgery of the spine}

Surgical techniques are advancing rapidly in the treatment of various spinal conditions. A great deal of research and clinical experience has been gained with the use of plates and screws throughout the spine, thereby affording immediate rigid fixation. Polymethylmethacrylate has been a very useful adjunct to conventional surgical techniques of stabilising the spine, particularly if involved with metastatic disease (Clark et al., 1984). A word of caution is in order when discussing the role of methylmethacrylate in spine surgery. This acrylic cement should only be used as an adjunct and the strict principles of conventional spine surgery must be adhered to. It should only be used to supplement a solid fusion construct and provisions for a bony arthrodesis should be made in all cases, except perhaps in the case of a metastatic lesion in a patient with a very limited life expectancy.

Anaesthesia techniques have greatly advanced our ability to effectively operate on spinal patients. The use of fiberoptic bronchoscopes to safely perform intubations in patients with spinal cord injury, as well as performing these intubations with the patient awake, has been a major advance. Spinal cord 
monitoring techniques utilising sensory evoked potentials have given us a means of monitoring the status of the spinal cord inter-operatively. Although these techniques are largely in the experimental and developmental stages at this time, they hold great promise for the future. Not only do these techniques have a potential of warning the surgeon that neural compromise may be occurring during the procedure, they also may be utilised to let the surgeon know that an adequate decompression has been achieved.

Intraoperative ultrasonography is a very useful technique. It has been largely used to determine the adequacy of tumour resection at the time of surgery, but we have also found it very helpful in determining whether we have achieved an adequate reduction of a traumatic spine deformity. Conventional interoperative radiographic studies are often misleading, particularly in the thoracolumbar region and ultrasound has been a major advance.

\section{Conclusions}

The orthopaedic surgeon plays a major role in the management of traumatic and other deforming conditions of the spine. There have been great strides in the treatment of these problems.

Neuroradiographic techniques allow safe and accurate diagnosis of spinal pathology. Magnetic resonance imaging has been a major advance in the area of noninvasive diagnosis. Further development of computer techniques will undoubtedly allow us to reconstruct and visualise the spine in a threedimensional manner.

Anaesthetic techniques of intubation and intraoperative monitoring and control of vital signs, have resulted in decreased bleeding, better visualisation for the surgeon, and less morbidity. Intraoperative spinal cord monitoring allows the surgeon to instantaneously monitor the status of neurologic function. Although this technique is in the experimental stage, it holds great promise for future development.

Surgical techniques and stabilisation devices have undergone a significant evolution. In vitro biomechanical testing as well as in vivo clinical studies will lead to further refinement in this area.

Through further research and development in all of the facets, we will learn much more about the spine and this will greatly enhance our ability to safely and effectively manage traumatic and deforming conditions.

\section{References}

Clark CR, Keggi KJ, PANJABI MM 1984 Methalmethacrylate stabilization of the spine. Fournal of Bone and foint Surgery 66A:40-46

GARFIN SR, BotTE MJ, CENTENo RS, et al. 1985 Osteology of the skull as it affects halo pin placement. Spine 10:696-698, Oct

KEAN DM, WORTHINGTON BS 1983 Craniovertebral junction pathology: Assessment by NMR. American fournal of Neuroradiology 4:232-233

KRAG M, Beynnon B, Gill K, et al. 1986 A new halo vest: Initial clinical experience and comparison to standard halo vests. Presented at the Cervical Spine Research Society Annual Meeting, December

PierCe DS, Nickel VH 1977 The total care of spinal cord injuries. Little, Brown and Co., Boston

SPETZLER RF, ZABRAMSKI JM, KAUFFMAN B 1985 Clinical role of magnetic resonance imaging in the neurosurgical patient. Neurosurgery 16:511-524 